\title{
MEASUREMENT AND CORRECTION OF LINEAR EFFECTS IN THE RHIC INTERACTION REGIONS
}

\begin{abstract}
V. Ptitsyn, J. Cardona, F. Pilat, BNL, Upton, USA Abstract

A considerable amount of betatron coupling in RHIC was found to originate from the interaction region (IR) triplets. A series of dedicated beam studies was performed with the goal of measuring the coupling errors in the IR triplets. The studies used two different methods: local interaction region orbit bumps and the action jump trajectory analysis. Availability of independently powered skewquadrupole corrector elements in the triplets allow local, beam-based coupling correction in the RHIC interaction regions using the analysed beam data. We also discuss plans for linear IR correction in the RHIC year 2001 run.
\end{abstract}

\section{INTRODUCTION}

At the beginning of the RHIC 2000 run one of the main aspects of the accelerator work was to ensure that the accelerator optics corresponds to what it is supposed to be according to the design. This work included the measurements of beta-functions, dispersion function as well as the search for the possible optics errors using difference orbit analysis. The analysis of the first turn beam trajectory with difference orbit method revealed clear sources of the betatron coupling in the interaction regions in the both RHIC rings. The example of what was seen is shown in Figure 1. The horizontal beam trajectory (on the first turn) is excited using a single dipole kick. After passing the interaction regions the clear trajectory oscillations are excited in the other (vertical) plane.

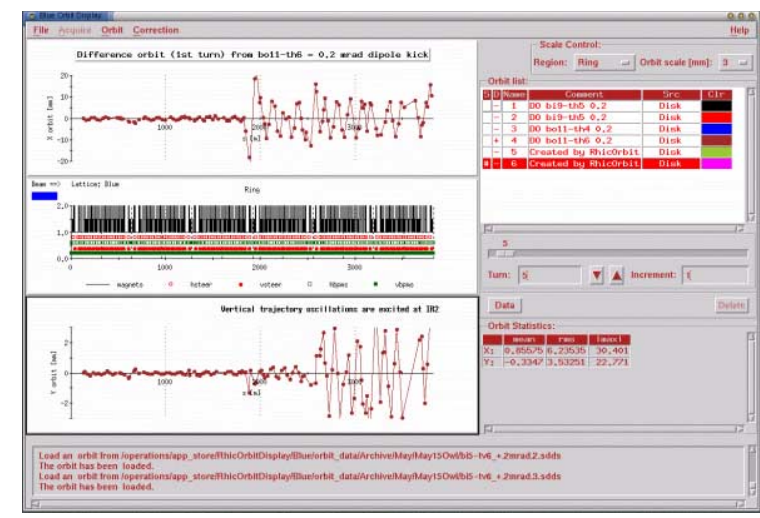

Figure 1: The betatron coupling sources at IRs were observed during the difference orbit RHIC optics mesurements.

With the circulating beam the considerable betatron coupling was measured (with $\Delta Q_{\min } 0.06$ )) and successfully corrected using the global coupling correction system with the skew-quadrupole correctors distributed in the ring arcs
[1]. But since we knew that the most of the coupling came from the interaction region it was decided to evaluate the coupling contribution from each interaction region and to consider applying the local correction. The interaction region triplets have the skew corrector elements installed among them that could be used to correct coupling right in the place of the error source.

To measure the value of the IR coupling error two techniques have been used independently. One of them was based on applying the closed orbit bumps at the interaction region triplets and looking at the closed orbit rms excited at the other plane. The second method, which we called orbit action jump method, utilized the single dipole kicks to excite the large beam trajectory oscillations at the first turn and looked at the change of the amplitude (action) of the trajectory oscillations in the opposite plane after every interaction region.

\section{MEASUREMENTS USING CLOSED ORBIT BUMPS}

The closed orbit bumps based on 3 dipole correctors were applied around the IR triplets. The value of the excited closed orbit rms in the plane opposite to the bump plane is proportional to the bump amplitude and to the value of the coupling error. Since we have the local skew quadrupole corrector inside the IR triplet and the betatron phase advance over the triplet is small, the corrector strength can be calculated to reduce or totally eleminate the orbit excitation in the opposite plane.

The measurements were done in the 3 interaction regions (IR2,IR6,IR8) in both RHIC rings (Blue and Yellow rings). In these IRs we had observed the strong sources of the betatron coupling. Also these are the IRs containing the experiment detectors and the local coupling compensation there should help us to improve the quality of the orbit bumps used to optimize the beam collision and improve the luminosity. The closed orbit bumps with amplitude from $5 \mathrm{~mm}$ to up to $60 \mathrm{~mm}$ (at some triplets) were apllied and the excited orbit rms in the ring arcs was measured as well as the shift of the betatron tunes. Doing so detailed bump scan we wanted also to get the information about nonlinear field harmonics in the triplets [2].

The presence of coupling errors excites the orbit rms in the plane opposite to the plane in which the bump was applied. The figures $(2,3)$ show the measured dependence of the rms orbit excited in the plane opposite to the bump plane versus bump strength. The sign of the rms orbit was choosen by looking at the sign of the orbit measured on the several BPMs in the arcs where according the model simulation we could expect positive orbit measurement in the 
case if the coupling error produced in the given IR triplet has positive sign. The linear slope of the measured curves at the region of the low bump amplitudes is proportional to the value of the coupling error.

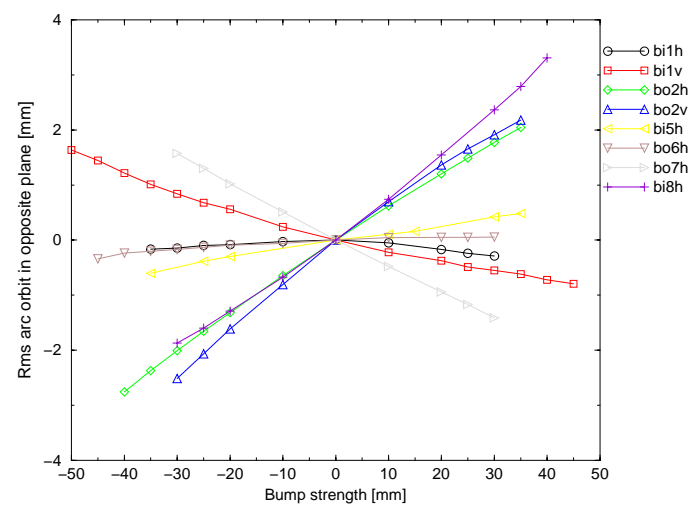

Figure 2: Orbit rms measured in the opposite plane versus the bump strength in Blue ring.

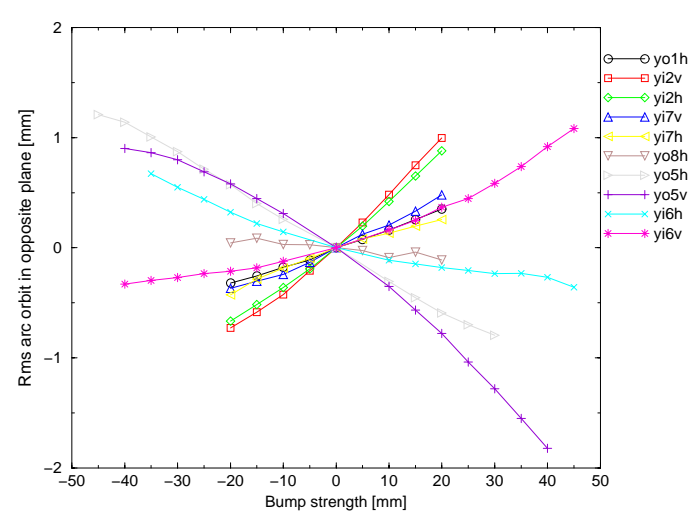

Figure 3: Orbit rms measured in the opposite plane versus the bump strength in Yellow ring.

Using the calculated slope of the measured curves and beta-functions at the skew quadrupole corrector place we can also extract the corrector strength neccesary to put the curve slopes to 0 , thus correcting for the coupling effect. The Table 1 lists the calculated skew corrector strength. The strongest coupling sources are at the Blue ring triplets at IR2 and IR8. The quadrupole rolls to produce the measured coupling effect at these triplets should be few mrad.

Table 1: Calculated IR skew corrector strengths.

\begin{tabular}{llll}
\hline \hline IR & $\begin{array}{l}\text { Triplet } \\
\text { Blue/Yellow }\end{array}$ & $\begin{array}{l}\text { Blue corr.str. } \\
\left(10^{-3} 1 / \mathrm{m}\right)\end{array}$ & $\begin{array}{l}\text { Yellow corr.str. } \\
\left(10^{-3} 1 / \mathrm{m}\right)\end{array}$ \\
\hline IR2 & I1/O1 & $-0.22 \pm 0.16$ & 0.3 \\
& O2/I2 & $1.23 \pm 0.15$ & $0.76 \pm 0$ \\
\hline IR6 & I5/O5 & 0.39 & $-0.94 \pm 0.08$ \\
& O6/I6 & 0.12 & $\pm 0.36 \pm 0.02$ \\
\hline IR8 & O7/I7 & -0.84 & $0.36 \pm 0.04$ \\
& I8/O8 & 1.32 & -1.1 \\
\hline \hline
\end{tabular}

\section{ORBIT ACTION JUMP METHOD}

Linear coupling at the IR leads to change in the amplitude of the betatron oscillation in every arc of the ring. This is evident if graphs of action vs the longitudinal coordinate $\mathrm{s}$ are derived from the difference orbit. An example of such graphs can be seen in figure 4 . The action at each point in the ring is calculated from the two nearest bpm measurements

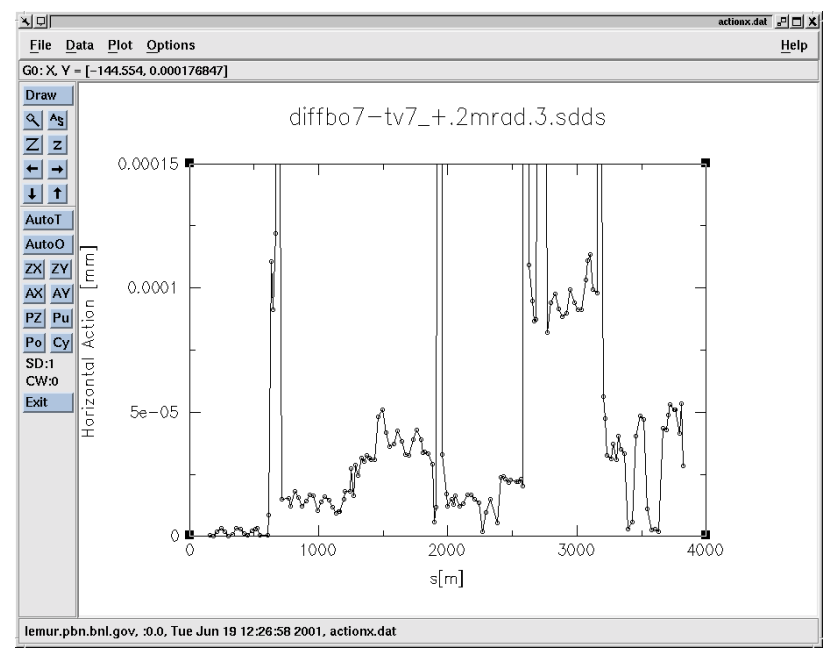

Figure 4: horizontal action of Orbit with kick in vertical plane.

If there were no coupling present in the ring the graphs of action vs s should be constant in the plane of the kick ( starting from the location of the kicker) and zero in the opposite plane. Thus, local linear decoupling is aimed to make the last graph as close to zero as possible.

In principle, the strength needed in the quadrupole correctors at any specific IR can be determined from either difference orbits with kicks in the arc immediately before the specific IR (principal strengths) or difference orbits with kicks in any previous arc different to the arc immediately before the specific IR (secondary strengths).

Principal strengths can be found by directly extracting the value of the corresponding induced action due to coupling $\left(J_{\text {coup }}\right)$ from the graphs of action vs s and using:

$$
S k_{\text {corr }}=-\frac{\sqrt{2 J_{\text {coup }}}}{y_{\text {corr }} \sqrt{\beta_{\text {corr }}}}
$$

here, $y_{\text {corr }}$ is the beam position in the plane of kick (in this case the vertical plane) at the location of the skew quadrupole corrector and $\beta_{\text {corr }}$ is the beta function at the position of the skew quadrupole corrector.

Secondary strengths require an additional step to calculate the $J_{\text {coup }}$ :

$$
\begin{aligned}
\sqrt{J_{\text {coup }}=} & J_{\text {after }}+J_{\text {before }}- \\
& 2 \sqrt{J_{\text {after }}} \sqrt{J_{\text {before }}} \cos \left(\varphi_{\text {after }}-\varphi_{\text {before }}\right)
\end{aligned}
$$


where $J_{\text {before }}$ is the action immediately before the IR, $J_{\text {after }}$ is the action immediately after the IR and $\varphi_{\text {before }}$ and $\varphi_{\text {after }}$ are the corresponding phases.

More than 40 Difference orbits with kicks at different positions in the ring have been analyzed and the strengths calculated at each IR leading to the following table:

\begin{tabular}{ccc} 
Table 2: Quadrupole Strengths for Blue Ring \\
\hline \hline IR & Strength & Real Settings \\
\hline & $10^{-3}$ & $10^{-3}$ \\
\hline IR8 & $(0.67 \pm 0.07)$ & 0.5 \\
IR10 & $(1.0 \pm 0.15)$ & 1 \\
IR12 & $(0.18 \pm 0.026)$ & - \\
IR2 & $(0.99 \pm 0.14)$ & 1 \\
IR4 & $(0.63 \pm 0.06)$ & - \\
IR6 & $(0.6 \pm 0.15)$ & - \\
\hline \hline
\end{tabular}

Principal and secondary strengths for the IR6, IR8, IR10 were pretty consistent. IR4 has an acceptable dispersion but only when principal strengths are considered (values shown in table). IR6 and IR12 have relatively high dispersions even for principal strengths.

Significant gradients errors can make useless the determination of secondary strengths. The reason for that can be understood if we compare the change in the orbit slope for a gradient error $\left(\Delta x_{\text {grad }}^{\prime}\right)$ and the corresponding change due to coupling $\left(\Delta x_{\text {coup }}^{\prime}\right)$ :

$$
\begin{aligned}
\Delta x_{\text {grad }}^{\prime} & =\frac{\Delta k}{k} k x \\
\Delta x_{\text {coup }}^{\prime} & =k 2 \phi y
\end{aligned}
$$

Big gradient errors can appear for example when secondary power supplies are inverted as it happened in RHIC leading to a variation of the integrated strength $k$ of about $10 \%\left(\frac{\Delta k}{k} \sim 0.1\right)$. The horizontal position of the beam $x$ is usually several times smaller than the vertical position of the beam (kick in the vertical plane) and taking into consideration that the roll angle $\phi$ is of the order of $\operatorname{mrad} \Delta x_{\text {grad }}^{\prime}$ is about one order of magnitude bigger than $\Delta x_{\text {coup }}^{\prime}$.

The third column of table 2 shows the values to which the skew quadrupoles has been set until now (run 2001). There is reasonable agreement between the last values and the measured strengths. The initial settings of the strengths for the quadrupoles were adjusted by trial and error using the IR bump method to test for the effectiveness of the decoupling. The good agreement between the second and the third column is encouraging the IR group of RHIC to do the remaining corrections in 1 step by using directly the measured values of the strengths.

The analysis of the difference orbits to obtain the table 2 before have been done with a special program designed for this purpose. The program takes the difference orbit data and convert it to action data vs s. The action associated with each arc is calculated as the average of all actions obtained for such arc. Formulas 3 and 1 are applied at each interaction region to find the corresponding strengths and print out (to the screen and to a file) tables similar to 2

\section{POSSIBLE EXPLANATION OF THE PRESENCE OF STRONG COUPLING}

The strengths associated with every IR lead to quadrupole rolls of several mRad. This relatively big number can be attributed to an error in the alignment procedure of the quadrupoles. In such procedure, the angle of misalignment (angle between the vertical axis of the pipe and the vertical axis of the quadrupole) is determined and saved in the database with the name field angle. There are technical reasons to think that the current roll of each quadrupole is equal to twice its corresponding field angle.

In order to validate the previous hypothesis the strengths calculated from the RHIC model asumming roll angles equal to twice field angle are compared with the measured strengths in the following table:

Table 3: Measured Strengths vs Model

\begin{tabular}{ccc}
\hline \hline IR & Strength & Real Settings \\
\hline & $10^{-3}$ & $10^{-3}$ \\
\hline IR8 & $(0.67 \pm 0.07)$ & 0.63 \\
IR10 & $(1.0 \pm 0.15)$ & -1.9 \\
IR12 & $(0.18 \pm 0.026)$ & 0.4 \\
IR2 & $(0.99 \pm 0.14)$ & -1.9 \\
IR4 & $(0.63 \pm 0.06)$ & 0.82 \\
IR6 & $(0.6 \pm 0.15)$ & 0.61 \\
\hline \hline
\end{tabular}

There is good agreement only for IR8 and IR6 but no for the others. The strengths are calculated taking into account the rolls of all the six quadrupoles at each interaction region. Many combinations of these six rolls can lead to the same strength. therefore, it is risky to draw a conclusion about the real roll angles.

\section{SUMMARY}

Both methods used to calculate the skew quadrupole corrector strength show a good agreement and really compliment each other. During these year RHIC run we remeasured again the skew quadrupole corrector strength at the IR2, IR8 and IR10 triplets at the Blue ring using the orbit bump technique. The measured strengths are in good agreement with the results obtained at run 2000. Thus, we plan to activate all local skew quadrupole correctors in both RHIC rings according to calculations made in this paper.

\section{ACKNOWLEDGEMENTS}

We are grateful to Steve Peggs, Todd Satogata, and Dejan Trbojevic for valuable discussions, suggestions and help in the realization of the experiment.

\section{REFERENCES}

[1] The coupling correction system at RHIC: results for the run 2000 and plans for 2001, F. Pilat et al., these proceed. .

[2] Beam-based measurements of field multipoles in the RHIC low-beta insertions and extrapolation of the method to $\mathrm{LHC}$, J.-P.Koutchouk, F.Pilat, V.Ptitsyn, these proceed. . 